\title{
ADEQUATE DISCRETE SAMPLING METHOD OF RANDOM VARIABLES OF TWO STAGE DRY CRUSHING PROCESS AND ITS STOCHASTIC EQUATION
}

\author{
Murat Adambayev ${ }^{1}$, Alma Auezova ${ }^{1}$, Assel Adambayeva ${ }^{2}$, Algazy Zhauyt ${ }^{1,3}$ \\ ${ }^{1}$ Almaty University of Power Engineering and Telecommunication, Kazakhstan; \\ ${ }^{2}$ LLC Copper KZ - CA Almaty, Kazakhstan; \\ ${ }^{3}$ Satbayev University \\ adambaev_m@mail.ru
}

\begin{abstract}
The article deals with stochastic equations of functional eight dry 2-stage mineral product grinding major variables in the form of correlation and regression dependencies. The dependencies were obtained including the factors, worsening the correlation of variables in question. We propose the algorithm of discretization of random variables, enabling to obtain their adequate discrete data set.
\end{abstract}

Keywords: grinding, autocorrelation, $\mathrm{T}$ implementation, discretization interval, regression equations.

\section{Introduction}

Is identification of static relationships of multidimensional stochastic controlled object on the basis of obtaining its eight variables adequate discrete data set? Identification of the mathematical model structure and parameters determination is a basic primary stage of synthesis of the automatic control system for any technological process [1]. There are known methods of analytical and experimental measuring the characteristics of controlled object properties [2;3]. Application of analytical research methods for identification of today's complex industrial multidimensional controlled objects, characterized by a multiplicity of interrelated variables seems rather difficult. Popular experimental methods on definition of properties are related with sending standard signals to a feeding part of the mill (step, harmonic, etc.). In recent years, there are widely used statistical research methods for identification of multi-loop controlled objects [4;5]. Random signals generated by a dry crushing machine in real working conditions are being caused by the change of raw material characteristics, ageing and deterioration of equipment, faulty operation of measuring devices, etc., thus preconditioning the stochastic nature of the relationship between input and output variables of the controlled object [6;7]. These relations are presented as correlation and dispersion relations, regression equations, etc. Full description of controlled object properties is seen on spectral densities of random input and output signals [8]. Thus, the problem of identification of static controlled object characteristics comes down to estimation of coefficient evaluation by equation formulas describing the object in question according to random realizations of input and output variables in normal operation conditions $[9 ; 10]$.

\section{Materials and methods}

The main requirement claimed to the automatic control system of the mill is in stabilization of loading singly its two chambers on the level that depends on hardness of the original ore fed.

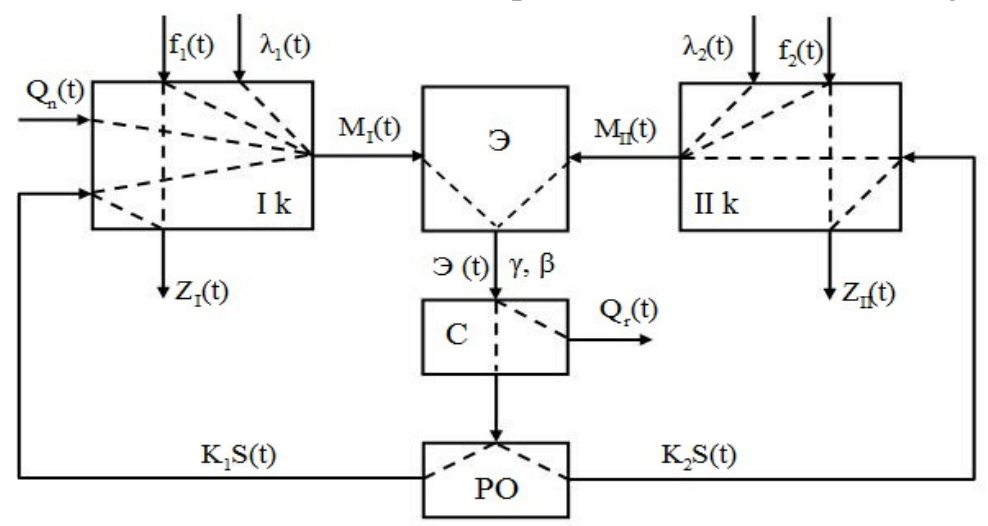

Fig.1. Object variables interaction: I k - coarse grinding chamber; II k - fine grinding chamber; $\ni$ - elevator; $\mathrm{C}$ - separator; PO-distributing trough (slide damper) 


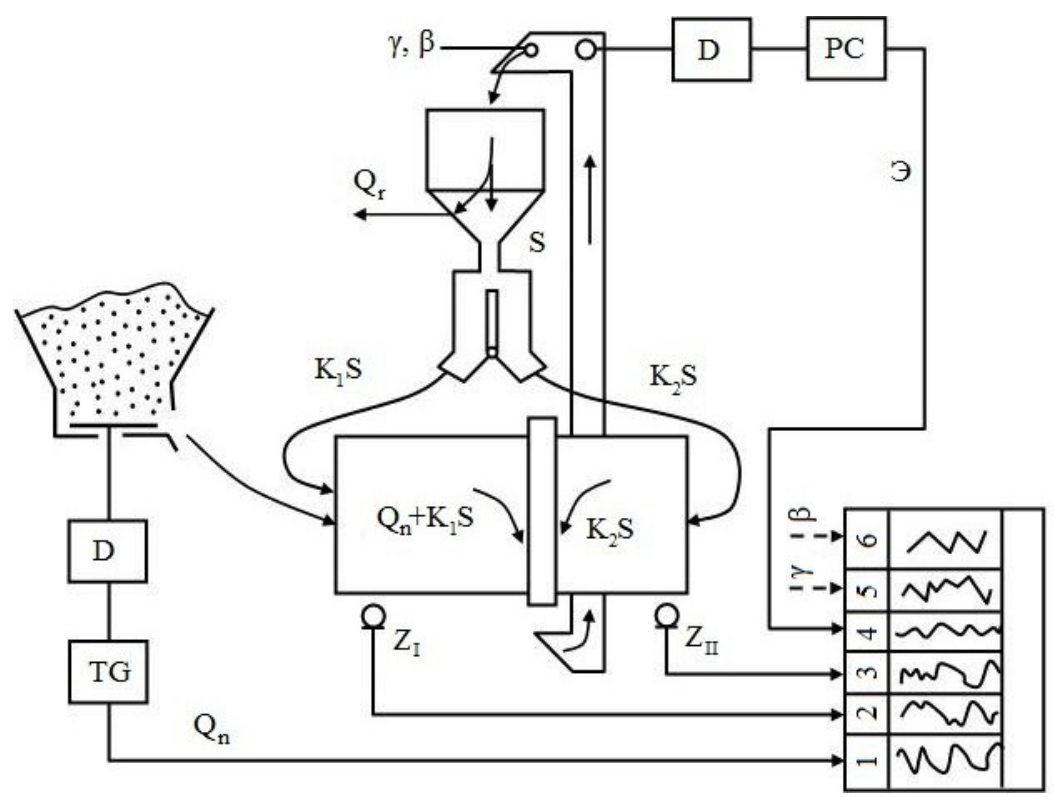

Fig.2. Mill variables registration scheme: $\mathrm{D}$ - engine; $\mathrm{TG}$ - rate generator; $\mathrm{PC}$ - power converter; Э - elevator

The output variables of two chamber filling, awaiting regulation $M_{I}(t), M_{I I}(t)$, are being controlled by intermediate controlled values (sound signals of chambers) $Z_{I}(t)$ and $Z_{I I}(t)$ [1], they are: the charge ore size $Q_{n}(t)$ is a control factor of a coarse grinding chamber loading level. The control factor for a fine grinding chamber is a part of circulating load $K_{2} S(t)$ that is redistributed into this camera by the distribution unit $(D U)$. The charge more for a fine grinding chamber is the ore sorted out by the separator $S$. The size of $K_{1} S(t)$ is the main signal for the coarse grinding chamber. Uncontrolled signals causing oscillations of output variables are divided into two types. One type affects both on $M_{I}(t)$, $M_{I I}(t)$ and $Z_{I}(t), Z_{I I}(t)-f_{I}(t), f_{I I}(t)$, the other $\lambda_{I}(t)$ and $\lambda_{I I}(t)$ - only on output variables (see Fig.1). Stabilization of controlled grinding process coordinates can be carried out by using the automatic control system, which development requires knowledge of static and dynamic characteristics of the mill. It has become possible after installing the automatic control of two chambers and overall loading [2-3], allowing to monitor such basic variables of the mill as the loading level of large and fine grinding chambers and the overall mill charging by capacity of the bucket elevator electric drive. In addition to the studies we also monitored a plate feeder performance by measuring the frequency of its rotation. The percentage of sorted out tailings and finished grade we determined by hand sampling using granulomere characteristics of the ground product at the mill feeder discharge. Figure 2 shows a simplified flow diagram of grinding with naming variables of the object subject to control for obtaining static equations of the relationship: $Z_{I}(t)$ - noise of coarse grinding chamber that displays the loading level of the chamber. It depends on the values of the two feed flows - base ore flow $Q_{n}(t)$ and a part of flow of circulating load $K S(t)$. The latter is regulated by the position of a distributing trough. Therefore, we have the following dependence:

$$
Z_{I}(t)=f\left[Q_{n}(t), K S(t)\right],
$$

where $Z_{I I}(t)$ - noise of the fine grinding chamber is a signal that displays the load level of the chamber that depends on another part of circulating load $[(1-K) S(t)]$ :

$$
Z_{I I}(t)=f[(1-K) S(t)],
$$

In the experiment, the value $K$ showing a degree of redistribution of circulating load between the milling stages remained unchanged. With this in mind, expressions Eq. (1) and Eq. (2) will take on form:

$$
\begin{gathered}
Z_{I}(t)=f\left[Q_{n}(t), S(t)\right], \\
Z_{I I}(t)=f[S(t)],
\end{gathered}
$$


where $\ni$ - bucket elevator gross loading with the ground product, defined by the total loading of two chambers and consisting of the sum of two variables:

$$
\ni(t)=S(t)+Q_{r}(t)
$$

where $S(t)$ - circulating load;

$Q_{r}(t)$ - weight of a newly formed finished product;

$\beta$ - off-test product content for a preset mill discharge weight determined by the size characteristics.

Then we have the following relation:

$$
S(t)=\beta(t) \cdot \ni(t),
$$

where $\gamma$-finished product content produced for the given moment $T_{I}$ and a preset mill discharge weight determined by the size characteristics.

Therefore, we have the following relation:

$$
Q_{r}(t)=\gamma(t) \cdot \ni(t)
$$

Adding expressions Eq. (6) and Eq. (7), we obtain:

$$
S(t)+Q_{r}(t)=\beta(t) \cdot \ni(t)+\gamma(t) \cdot \ni(t)=\ni(t)
$$

because

$$
\gamma(t)+\beta(t)=1
$$

Expression Eq. (8) is equal to expression Eq. (5), so the material balance on the elevator is adhered. To determine the main stochastic relations between the mill variables we have obtained the following random realizations: $Z_{I}(t)$ - sound signal of the coarse grinding chamber (automatic entry); $Z_{I I}(t)$ - sound signal of the fine grinding chamber (automatic entry); $Q_{n}(t)$ - size of the power source (automatic entry); Э $(t)$ - bucket elevator load (automatic entry); $\beta(t)$ - change of tailings per unit of mill discharge weight (determined by size characteristics); $\gamma(t)$ - change of newly formed finished product in the unit of mill discharge weight (determined by the size characteristics); $S(t)$ - circulating load (defined by expression (see Eq. (6))); $Q_{r}(t)$ - size of the finished product (determined by expression (see Eq. (7))). Implementation of random variables has been recorded on a six-point potentiometer (see Fig.2), at this the parameters $Z_{I}(t), Z_{I I}(t), Q_{n}(t), \ni(t)$ were recorded automatically on a strip chart and the curves of variables implementation $\beta(t), \gamma(t), S(t)$ and $Q_{r}(t)$ were obtained in the result of simultaneous imposition of these variable discrete values on the diagram obtained after appropriate processing the data of the laboratory granulomere sample analysis. For a statistical processing of the obtained implementations it is necessary to present them in a discrete form for using the appropriate modern computer software. This requires selecting on a reasonable basis a desired time of duration of $T$ implementation and the interval of sampling or quantization $\Delta t$. To select $T$ there were obtained autocorrelation functions $R_{X X}(\tau)$ of all 8 variables of the object (see Fig.3). The dead time of each of them is taken to be equal to a time segment, beyond which the inequality holds true [4]:

$$
R_{X X}(\tau) \leq 0.05 \cdot R_{X X}(0) .
$$

The required duration of random variables of the object (mill) is taken as being equal to the maximum amount of dying down time $\tau_{\max }$.

But due to the formula suggested in [5] for $T$ determination we shall obtain:

$$
T=10 \tau_{\max } \text {. }
$$

$T=10 \cdot 22 \min =220 \min \approx 4$ hours, Because $\tau_{\max }=22 \min$ (see Fig. 3 ).

In this work the values of random variables realization were being recorded during 10 hours, of which we have chosen a central block of the oscillogram with the established regime with 4 hours duration. We have excluded the initial and final sections of the oscillogram, where, as we believe, the instability of the milling process takes place (see Fig. 4). The sampling interval or the interval of oscillograms quantization was selected on the basis of applying a sampling theorem (Kotelnikov's 
theorem) for the highest frequency variables. According to the Kotelnikov's theorem, the continuous function $X(t)$ with a limited frequency spectrum $\Delta f=f_{\max }-f_{\min }$ can be discretized by sequence $X_{1}, X_{2}, \ldots X_{n}$ with the interval equal to:

$$
\Delta t=1 / 2 \Delta f .
$$

The number of $\mathrm{n}$ intervals on the oscillogram is determined by the formula:

$$
n=T / \Delta t=2 t \cdot \Delta f \text {. }
$$

The random realizations curve is used for measuring maximum and minimum periods of oscillations, also they are used for determination of the difference of frequencies $\Delta \omega=\omega_{\max }-\omega_{\min }$ from the formula:

$$
\Delta \omega=2 \pi\left(\frac{1}{T_{\text {min }}}-\frac{1}{T_{\text {max }}}\right)=2 \pi \frac{T_{\text {max }}-T_{\text {min }}}{T_{\text {max }} \cdot T_{\text {min }}} .
$$

Adopting $\Delta \omega \approx \Delta f$ and substituting the data $\Delta \omega$ in the formula Eq. (12), we determine the $n$ value from the formula:

$$
n=\frac{4 \pi \cdot T \cdot\left(T_{\max }-T_{\min }\right)}{T_{\max } \cdot T_{\min }} .
$$

For the selected realization of the variable $Z_{I}(t)$ there exists (see Fig. 4):

$$
T=240 \mathrm{~min}, T_{\max }=60 \mathrm{~min}, T_{\min } 20 \mathrm{~min} \text {. }
$$

Then, from formula Eq. (14) we can define:

$$
n=\frac{4 \cdot 3.14 \cdot 240 \cdot(60-20)}{60 \cdot 20}=100.5
$$

Increasing by $15 \%$ the obtained number $\mathrm{n}$ for recording the instability of the frequency response of the curve we have: $n=100.5 \cdot 1.15=1162$.

Finally we adopt the number $\mathrm{n}$ as being equal to 120 . Then the sampling interval will be equal to:

$$
\Delta t=240 / 120=2 \mathrm{~min} \text {. }
$$

\section{Results and discussion}

This interval of sampling is adopted for making a discrete set of data of the mill variables. We have obtained discrete values of random realizations of eight mill variables with a 2 min. interval of quantization (in 120 values), which are used for calculation of math relationships [6]. The assigned task was solved on the computer with the use of a standard software program SPSS-18. The results of experimental data calculation on the computer are shown in Table 1.

The analysis of the results of pair correlation speaks about one of their features - correlation coefficients between the variables of two-stage dry grinding with integral separation have relatively low values.

Table 1

Pair correlation of object variables

\begin{tabular}{|c|c|c|c|c|c|c|c|}
\hline \multirow{2}{*}{ Dependence } & \multicolumn{4}{|c|}{$\boldsymbol{y}=\boldsymbol{a}+\boldsymbol{b} \boldsymbol{x}$} & \multicolumn{4}{c|}{$\boldsymbol{y}=\boldsymbol{a}+\boldsymbol{b} \boldsymbol{x}+\boldsymbol{c} \boldsymbol{x}^{\mathbf{2}}$} \\
\cline { 2 - 8 } & $\mathrm{a}$ & $\mathrm{b}$ & $f$ & $\mathrm{a}$ & $\mathrm{b}$ & $\mathrm{c}$ & $\eta$ \\
\hline$Z_{1}=f\left(Q_{n}\right)$ & 116.9 & -0.18 & -0.19 & 32.6 & 1.2 & -0.008 & 0.39 \\
\hline$Z_{1}=f(S)$ & 125.5 & -0.71 & -0.25 & 144.2 & -2.3 & 0.027 & 0.31 \\
\hline$Z_{I I}=f(S)$ & 142.7 & -2.3 & -0.78 & 149.5 & -2.9 & 0.01 & 0.80 \\
\hline$Э=f(S)$ & 11.1 & 0.8 & 0.96 & - & - & - & - \\
\hline$\gamma=f\left(z_{I}\right)$ & 59 & 0.13 & 0.28 & 58.4 & 0.17 & -0.002 & 0.28 \\
\hline$\gamma=f\left(z_{I I}\right)$ & 45.5 & 0.35 & 0.75 & 50.3 & 0.17 & -0.001 & 0.76 \\
\hline$\gamma=f(\ni)$ & 106.7 & -1.2 & -0.90 & 118.7 & -2.3 & 0.017 & 0.94 \\
\hline
\end{tabular}




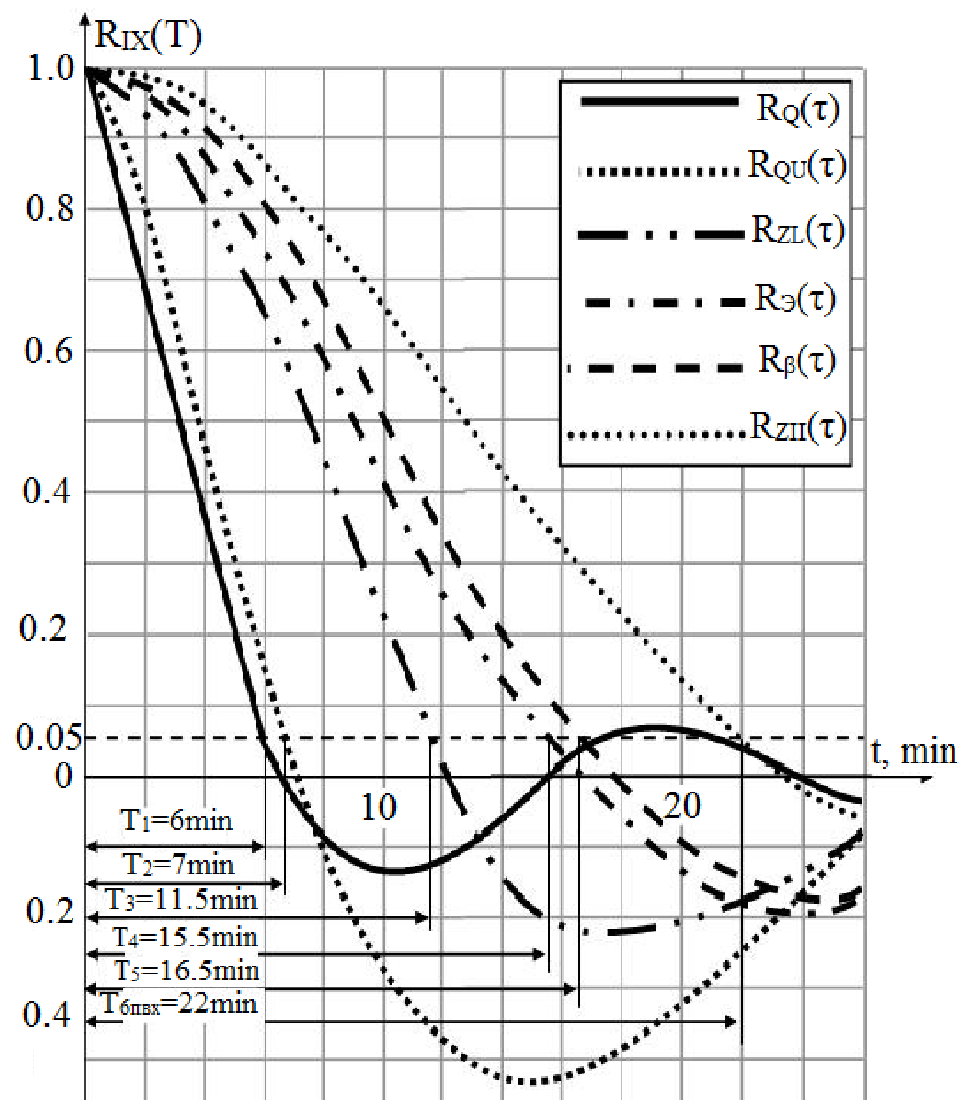

Fig. 3. Variables autocorrelation functions

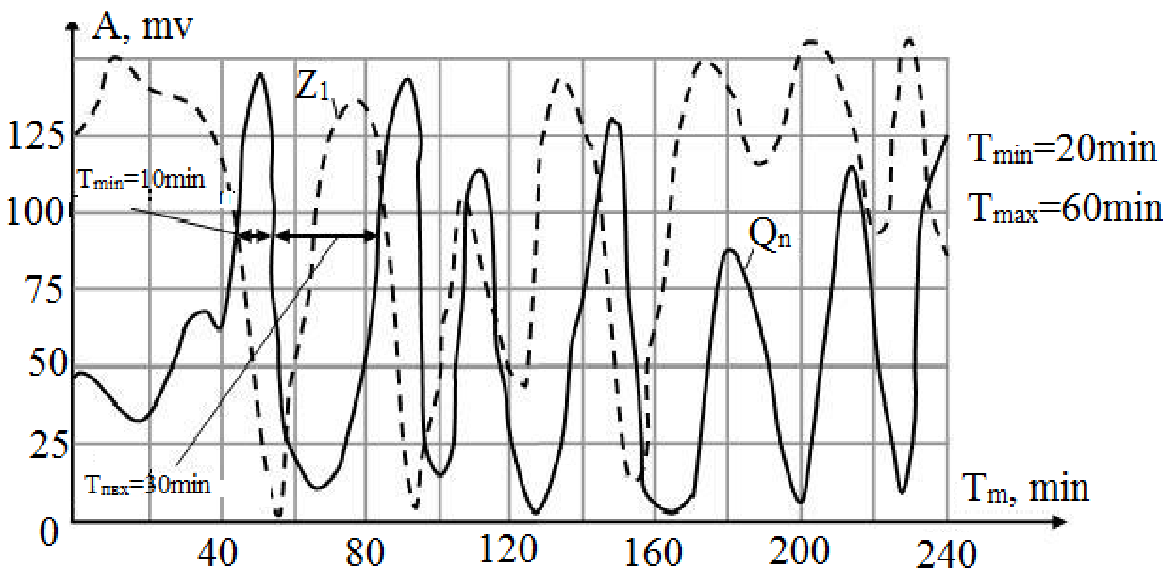

Fig. 4. Random realizations $Q_{n}(t), Z_{I}(t)$ (the rest implementations are not shown here)

Reasons of pair bonding low correlation:

1. A two-chamber ball mill is the object with many inputs and outputs that influence each other on basic and cross-technological channels. The link between the input and output is broken by an unaccounted variable (variables) that significantly effects on the output size of the signal;

2. The presence of nonlinear relation between variables of input and output signals leads to reduction of the correlation coefficient obtained under our assumption about the linearity of the link;

3. The object in question is inertial. Between its input and output variables there are large lags that also distort a stochastic relation of the desired dependences.

Development of a mathematical model of the mill in the form of multiple regression equations is the solution of the problem, when the value of output coordinate is determined by the values of many input signals [7]. It should be pointed out that a sound response $Z_{I}(t)$ of the coarse grinding chamber 
depends on the chamber ore feeding level which, in turn, it is determined by the sum of two flows the base ore flow $Q_{n}(t)$ and a part of circulating load flow coming into this chamber $K S$. It means that this sound response $Z_{I}(t)$ depends at least on two input signals. So, it is necessary to determine the regression of three variables $Z_{I}(t), Q_{n}(t), S_{I}(t)$, i.e. to find out the $Z_{I}=f\left(Q_{n}, S\right)$ form dependence.

Similarly, the signal quantity of circulating load $S$, the percentage of the newly formed ready class $\gamma$ and substandard class $\beta$ per unit of weight of the mill discharge depend mainly on the feed level of both chambers. One needs to define regression equations relationship of the form:

$$
S=f\left(Z_{I}, Z_{I I}\right) ; \gamma=f\left(Z_{I}, Z_{I I}\right) ; \beta=f\left(Z_{I}, Z_{I I}\right) .
$$

Factors influencing the mill performance regarding the finished product $Q_{r}$ are a level of feeding the chamber with ore, as well as signal quantity of a circulating load in the system [8]. Regression relationship takes the form:

$$
Q_{r}=f\left(Z_{I}, Z_{I I}, S\right) .
$$

In order to measure the effect of nonlinearity on correlation of paired connections one should know the form of this nonlinearity that we estimate in the following way. We approximated the desired paired associations by standard dependencies: linear $y=a+b x$; power $y=a x^{b}$; logarithmic $y=a+b \ln x$; hyperbolic $y=a+b / x$; parabolic $y=a+b x+c x^{2}$.

The correlation ratio between the variables is estimated by the correlation ratio $\eta$. The maximum value of the latter is determined by way of comparing the obtained correlation dependencies subject to the absolute value of the same pair of variables, calculated on the assumption that between them there is possible any of the above dependencies. The correlation law, for which we received the maximum value of the correlation ratio, approximates most closely the form of nonlinear relation between the variables [9]. Due to the fact that large correlation coefficients were obtained for linear and parabolic forms of connection, the data obtained for other dependencies or laws in Table 1 is not given. Influence of mill dynamic properties on the degree of stochastic connection between the variables is determined by the method that is the torque reading or quantization of input variable shifts by $\Delta t_{1}, \Delta t_{2}$, time intervals from the moment of registration of the output variable [10].

Due to the fact that the curves of valuable implementation have a complicated nature it is impossible to estimate lag quantities between the input and output variables by direct measurement by the oscillogram. In the paper we were making the assessment of shift quantities $\Delta t_{1}, \Delta t_{2}$ by mutualcorrelation functions (see Fig. 5). Each of values $\Delta t$ is equal to time lag, where a mutual correlation function $R_{y x}(\tau)$ attains the maximum.

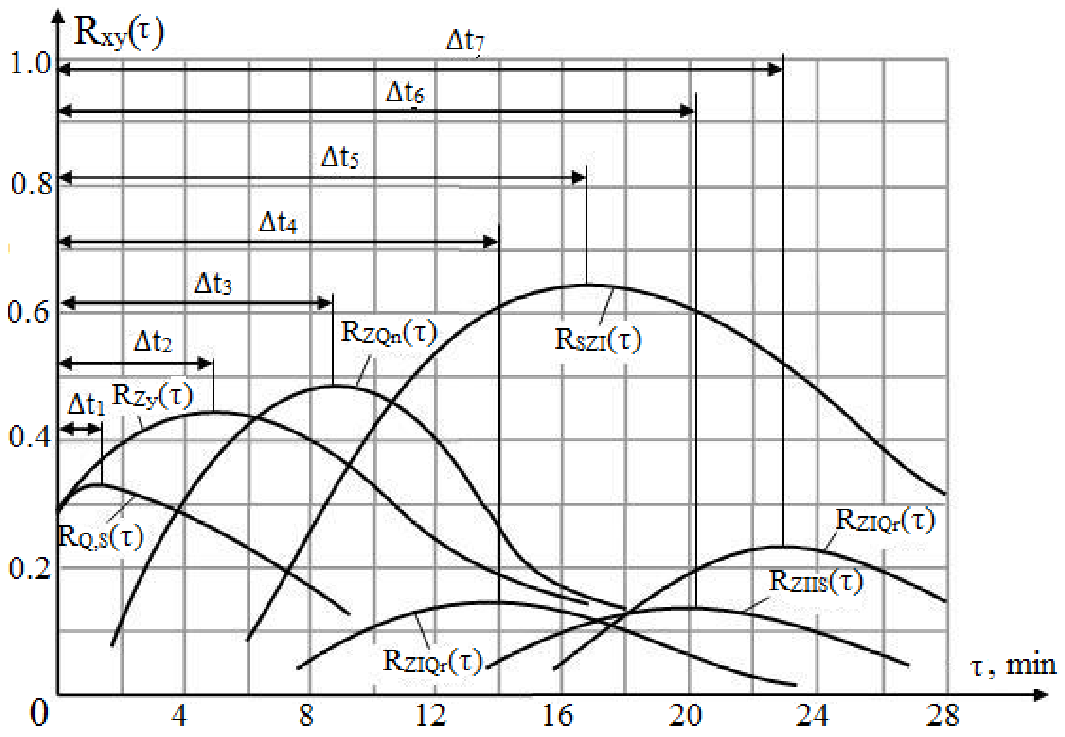

Fig. 5. Cross-correlation functions of valuables 
Regression relationship equations of mill variables

Table 2

\begin{tabular}{|c|c|c|c|c|}
\hline Equation of regression & $\boldsymbol{f}_{\mathbf{1 2}}$ & $\boldsymbol{f}_{\mathbf{1 3}}$ & $\boldsymbol{f}_{\mathbf{2 3}}$ & $\boldsymbol{R}$ \\
\hline$Z_{I}=144.9-0.26 Q_{n}-0.95 S$ & -0.41 & -0.51 & -0.25 & 0.74 \\
\hline$Z_{I I}=118.4+0.33 Q_{n}-2.15 S$ & 0.5 & -0.79 & -0.25 & 0.85 \\
\hline$\ni=62.8-0.11 Z_{I}-0.23 Z_{I I}$ & -0.25 & 0.75 & -0.18 & 0.84 \\
\hline$\gamma=20.7+0.2 Z_{I}+0.38 Z_{I I}$ & 0.28 & 0.75 & 0.18 & 0.86 \\
\hline
\end{tabular}
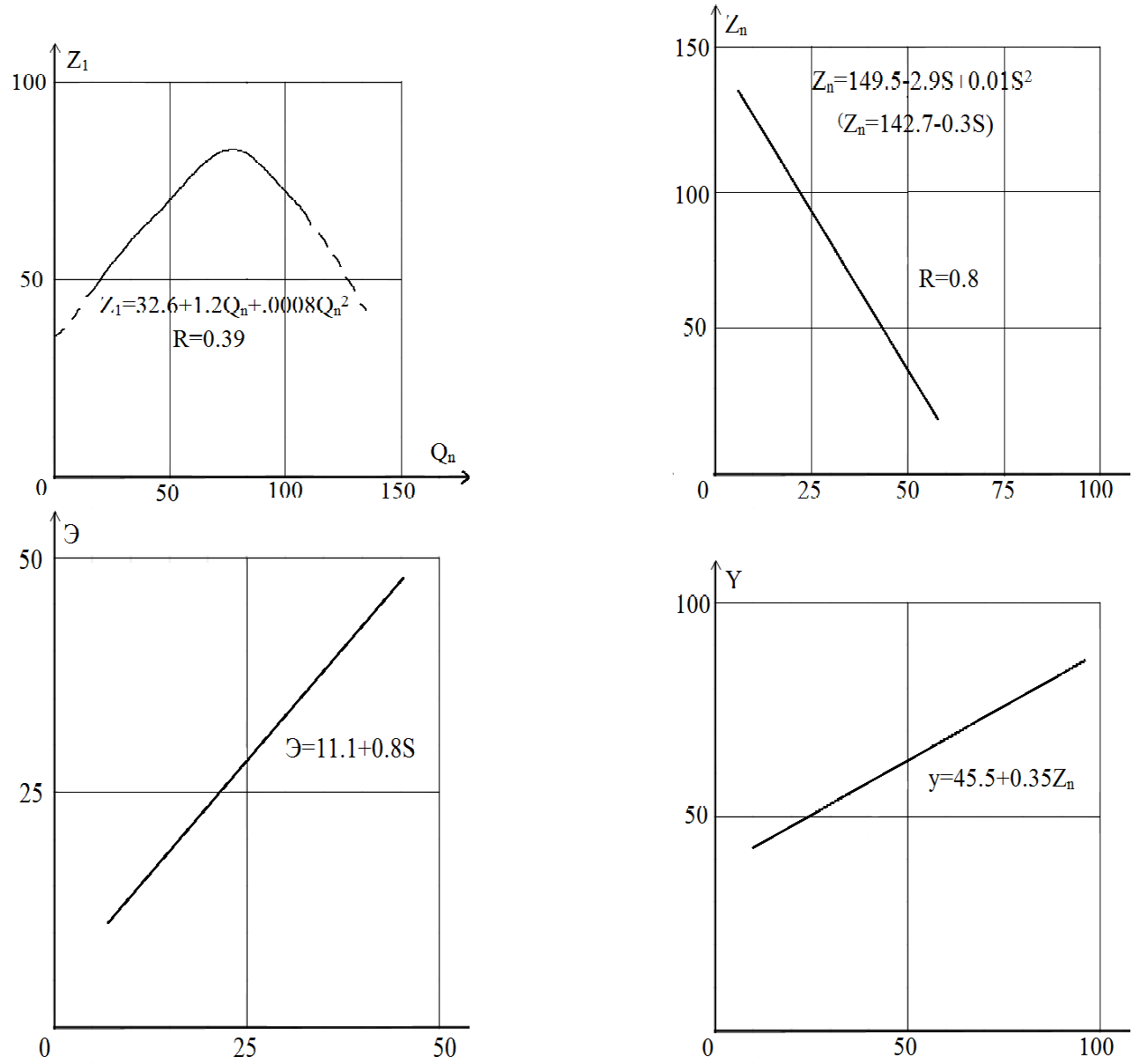

Fig. 6. Regression dependencies of mill parameters

The results of correlation dependences calculation on computing machines with explaining the causes that affect the correlation of the mill parameters are shown in Table 2. The analysis of the data obtained enables to conclude that static characteristics of the two-chamber ball mill are consistent with well-known foreign analogues. Some of regression laws are shown in Fig. 6. We used Student and Fisher's t-tests for estimating the reliability of the correlation coefficients: $a, b, c$ regression equation coefficients; $f$ coefficient of pair correlation; $f_{i j}$ coefficient of private correlation; $R$ total coefficient of correlation; $\eta$ correlation ratio.

\section{Conclusions}

1. Dry two-stage grinding process with joint separation of both chamber mill products is a multidimensional object of control, which basic variables are random temporal functions. To obtain equations of stochastic object variable relation (static identification) there is provided the algorithm of receiving adequate discrete set of data and using it in modern software products (computers).

2. We have received correlation and regression ratio of the object under research together with the factors affecting and distorting the correlation of stated equations (influence of unsuspected 
variable, of their connection forms on the value of the correlation coefficient, inertia and lag between input and output variables). There are provided quantitative correlation and regression equations of variable relation, which easily may synthesize a structure of control systems along different control channels.

\section{Acknowledgements}

The article was written on the research No. 747.Mon.GF.12.13 pursuant to program "Grant financing of scientific research projects for 2012-2014 (Research project supervisor prof. M. Adambayev). The authors express their gratitude to Muhammad Monowar Hossain Mahmood, scientific adviser, $\mathrm{PhD}$ in Management Sciences, University of Manchester, as well as to University temporary labor staff, Bs, Ms, PhD students of Department of Power Engineering and Process Automation.

\section{References}

[1] Hicks Ch. Fundamental Concepts in the Design of Experiment's. Translation from English Moscow, MIR, 1967, pp. 406-412.

[2] Lukomsky Y. N. Correlation theory and its application to the analysis of production. Gosstatizdat, 1961, pp. 324-333.

[3] Balakirev V. S., Dudnikov E. G., Tsirlin A. M. Experimental determination of dynamic characteristics of industrial objects of control. Energy, 1967, pp. 25-36.

[4] Hartmann K., Lezkyi E., Sheffer W. Planning an experiment in the study of technological processes. Translation from German, MIR, 1977, pp. 552-564.

[5] Adambayev M. D. Theory and practice of technical experiment in electric power industry. KazNTU, 2013, pp. 237-251.

[6] Protosenya A., Kulish S., Azbel Y. Mathematical methods and models in the planning and management of mining production. Nedra, 1985, pp. 288-297.

[7] Volgin V., Karimov R. Evaluation of correlation functions in industrial control systems. Energy, 1979, pp. 80-91.

[8] Yang S., Allison A. Measurement of traffic noise: Translation from English. A. N. Halacheva. Energoatomizdat, 1988, pp. 44-58.

[9] Meerov M. Multiply connected control systems. Nauka, 1965, pp. 384-401.

[10] Adambayev M. D., Abdikadirov N. N., Adilkhanov D. B., Dostanov D. A. Innovative patent No. 20963 of the Republic of Kazakhstan. "Method of automatic control of total load twocompartment separator mill the device for its implementation". Posted 16.03.2009, bull. No. 3 . 